\title{
INTERNATIONAL JOURNAL OF MEDICINAL MUSHROOMS
}

\author{
CONTENTS, VOLUME 21, 2019
}

Page Range of Issues - Issue 1: 1-103; Issue 2: 105-206; Issue 3: 207-309; Issue 4: 311-411; Issue 5: 413-521; Issue 6: 523-628; Issue 7: 629-734; Issue 8: 735-839; Issue 9: 841-942; Issue 10: 943-1050; Issue 11: 1051-1150; Issue 12: 1151-1247

\section{Issue 1}

In Vitro and In Vivo Inhibition of Helicobacter pylori by Ethanolic Extracts of Lion's Mane Medicinal Mushroom, Hericium erinaceus (Agaricomycetes)

Ge Wang, Xiumin Zhang, Susan E. Maier, Liping Zhang, \& Robert J. Maier

Immunomodulatory Activities of Polysaccharides from White Button Mushroom, Agaricus bisporus (Agaricomycetes), Fruiting Bodies and Cultured Mycelia in Healthy and Immunosuppressed Mice

Yang Liu, Dandan Zheng, Dinghe Wang, Ling Su, Qi Wang, \& Yu Li

Aqueous Extract of Wood Ear Mushroom, Auricularia polytricha (Agaricomycetes), Demonstrated Antiepileptic Activity against Seizure Induced by Maximal Electroshock and Isoniazid in Experimental Animals Gaurav Gupta, Sachchidanand Pathak, Rajiv Dahiya, Rajendra Awasthi, Anurag Mishra, Rakesh Kumar Sharma, Mohit Agrawal, \& Kamal Dua

Dissolution of Bioactive Components from Dried Fruiting Bodies of the Culinary-Medicinal Shiitake Mushroom, Lentinus edodes (Agaricomycetes), during Cleaning, Soaking, and Cooking Lina Zhu, Shulei Wang, Zhong Zhang, Shuai Zhou, Qingjiu Tang, Feihua Wu, \& Jingsong Zhang

Developing a Novel Two-Stage Process for Carotenoid Production by Cordyceps militaris (Ascomycetes)

Qianwang Zheng, Tao Wei, Yin Lin, Zhiwei Ye, Junfang Lin, Liqiong Guo, Fan Yun, \& Linzhi Kang

Effect of Environmental Conditions on Synnema Formation and Nucleoside Production in Cicada Flower, Isaria cicadae (Ascomycetes) Kuanbo Liu, Fen Wang, Guijun Liu, \& Caihong Dong

Radiocesium (137Cs) and Mineral Elements in Culinary-Medicinal Mushrooms from the Southern Outskirts of Kyiv, Ukraine Anna A. Grodzinskaya, Anatoliy I. Samchuk, Vitaliy B. Nebesnyi, \& Hanna Yu. Honchar

Shiitake Culinary-Medicinal Mushroom, Lentinus edodes (Agaricomycetes), Supplementation Alters Gut Microbiome and Corrects Dyslipidemia in Rats

Haseeb Anwar, Jan S. Suchodolski, Muhammad I. Ullah, Ghulam Hussain, Muhammad Z. Shabbir, Imtiaz Mustafa, \& Muhammad U. Sohail

Effects of Diets Supplemented with Medicinal Mushroom Myceliated Grains on Some Production, Health, and Oxidation Traits of Dairy Ewes Adriana Bonanno, Antonino Di Grigoli, Francesca Vitale, Giuseppe Di Miceli, Massimo Todaro, Marco Alabiso, Maria Letizia Gargano, Giuseppe Venturella, Felicia Ngozi Anike, \& Omoanghe Samuel Isikhuemhen

\section{$\underline{\text { Issue } 2}$}

Cancer without Pharmacological Illusions and a Niche for Mycotherapy (Review) Ivan V. Zmitrovich, Nina V. Belova, Mikhail E. Balandaykin, Margarita A. Bondartseva, \& Solomon P. Wasser

Fruiting Body Production of, and Suitable Environmental Ranges for, Growing the Umbrella Polypore Medicinal Mushroom, Polyporus umbellatus (Agaricomycetes), in Natural Conditions in Central Europe Vladimír Kunca \& Martin Pavlík 
Evaluation of the Antibacterial Activity of 75 Mushrooms Collected in the Vicinity of Oxford, Ohio (USA)

Faizule Hassan, Shuisong Ni, Taylor L. Becker, Christine M. Kinstedt, Jana L. Abdul-Samad, Luis A. Actis, \& Michael A. Kennedy

Effects of Concurrently Administered Aqueous Extract of Lingzhi or Reishi Medicinal Mushroom, Ganoderma lucidum (Agaricomycetes), and Lead Acetate in Rats 143

Michael T. Sobowale, Raymond I. Ozolua, Dickson O. Uwaya, \& Buniyamin A. Ayinde

Antioxidant-Rich Peptide Fractions Derived from High-Altitude Chinese Caterpillar Medicinal Mushroom Ophiocordyceps sinensis (Ascomycetes) Inhibit Bacterial Pathogens

Jigni Mishra, Rakhee Rajput, Kaushlesh Singh, Anju Bansal, \& Kshipra Misra

Monitoring Heavy Metals in Royal Sun Medicinal Mushroom, Agaricus brasiliensis (Agaricomycetes)

Eduardo Marin Morales, Bruna Letícia Baldissera, \& Dejanira de Franceschi de Angelis

Bioprospecting of Wild Mushrooms from India with Respect to Their Medicinal Aspects Foziya Khan \& Ramesh Chandra

Safety Evaluation of an Aqueous Extract of Termitomyces robustus (Agaricomycetes) in Wistar Rats

Eziuche Amadike Ugbogu, Emmanuel Iroha Akubugwo, Victor Chibueze Ude, Okezie Emmanuel, Nduka Ozoji Okomba, Cynthia Ibe, \& Ozioma Onyero

Mushroom Cultivation: An Illustrated Guide to Growing Your Own Mushrooms at Home Craig Soderberg

\section{Issue 3}

Chemical Compounds and Antioxidant Activity of Volatile Oil from the White Jelly Mushroom,

Tremella fuciformis (Tremellomycetes)

Wei Liu, Qing-jiu Tang, Yu-tian Wei, Le Han, Wei Han, Na Feng, \& Jing-song Zhang

Cultivation of Medicinal Mushrooms on Spruce Sawdust Fermented with a Liquid Digestate

from Biogas Stations

Adam Brezáni, Kateřina Svobodová, Ivan Jablonský, \& Pavel Tlustoš

The Potential Role of Medicinal Mushrooms in the Prevention and Treatment of Gynecological Cancers: A Review

Giuseppe Venturella, Paola Saporita, \& Maria Letizia Gargano

New Insight into Mycochemical Profiles and Antioxidant Potential of Edible and Medicinal Mushrooms: A Review Tahidul Islam, Kumar Ganesan, \& Baojun Xu

Engineering Aspects of Lingzhi or Reishi Medicinal Mushroom Ganoderma lucidum (Agaricomycetes) Biomass Submerged Cultivation in Bioreactors: A Review Marin Berovic \& Bojana Boh Podgornik

Medicinal Sulphur Polypore Mushroom Laetiporus sulphureus (Agaricomycetes) Plus Tiny Amounts of Essential Oils Decrease the Activity of Crohn Disease Bruno Donatini \& Isabelle Le Blaye

Pleurotus ostreatus and Laetiporus sulphureus (Agaricomycetes): Possible Agents against

Alzheimer and Parkinson Diseases Jasmina Ćilerdžić, Milica Galić, Jelena Vukojević, \& Mirjana Stajić

Analysis of Intraspecies Genetic Variability among Collections of Medicinal Red Belt Conk Mushroom, Fomitopsis pinicola (Agaricomycetes) Alla V. Shnyreva, Susanna M. Badalyan, \& Anastasia A. Shnyreva

Identification and Evaluation of Reference Genes for qRT-PCR Normalization in Sparassis latifolia (Agaricomycetes) Chi Yang, Lu Ma, Donglai Xiao, Zhenghe Ying, Xiaoling Jiang, \& Yanquan Lin 
Optimization of the Process for Producing Biomass and Exopolysaccharide from the King Tuber Oyster Mushroom, Pleurotus tuber-regium (Agaricomycetes), for Biotechnological Applications Comfort Olukemi Bamigboye, Julius Kola Oloke, Mike Burton, Joanna Felicity Dames, \& Agbaje Lateef

Role of Aqueous Extract of the Wood Ear Mushroom, Auricularia polytricha (Agaricomycetes), in Avoidance of Haloperidol-Induced Catalepsy via Oxidative Stress in Rats Xiaohong Liu, Rakesh Kumar Sharma, Anurag Mishra, Gopala Krishna Chinnaboina, Gaurav Gupta, \& Mahaveer Singh

Comparison of Chemical Compositions, Bioactive Ingredients, and In Vitro Antitumor Activity of Four Products of Cordyceps (Ascomycetes) Strains from China Jie Qiao, Yuying Shuai, Xin Zeng, Dayong Xu, Shengqi Rao, Huawei Zeng, \& Feng Li

Assessing the Bioavailability of Zinc and Indole Compounds from Mycelial Cultures of the Bay Mushroom Imleria badia (Agaricomycetes) Using In Vitro Models Katarzyna Kała, Agata Krakowska, Joanna Gdula-Argasińska, Włodzimierz Opoka, \& Bożena Muszyńska

Optimization of Cultivation Conditions of Lingzhi or Reishi Medicinal Mushroom, Ganoderma Iucidum (Agaricomycetes) for the Highest Antioxidant Activity and Antioxidant Content Hai-wei Lou, Xin-yue Guo, Xin-chao Zhang, Li-qiong Guo, \& Jun-fang Lin

Mitigating Acetaminophen-Induced Hepatotoxicity by Using a Water-Alcohol Extract of Phellinus caryophylli (Agaricomycetes) in a Murine Model Yogesh Bharat Dalvi, Ruby Varghese, Prasad Y. Lamrood, \& C.K.K. Nair

Methyl-Hispolon from Phellinus lonicerinus (Agaricomycetes) Affects Estrogen Signals in MCF-7 Breast Cancer Cells and Premature Aging in Rats Junzhi Wang, Huifang Lv, Beiyan Chen, Wenfeng Huang, Ailing Wang, Lanqing Liu, Haibo He, Jianfeng Chen, Shi Li, \& Wei-Qiao Deng

The Impact of Drying of Wild-Growing Mushrooms on the Content and Antioxidant Capacity of Water-Soluble Polysaccharides

Wojciech Radzki, Aneta Stawińska, Katarzyna Skrzypczak, \& Monika Michalak-Majewska

Thirteen-Week Oral Toxicity Evaluation of Erinacine A-Enriched Lion's Mane Medicinal Mushroom, Hericium erinaceus (Agaricomycetes), Mycelia in Sprague-Dawley Rats Li-Ya Lee, I-Chen Li, Wan-Ping Chen, Yueh-Ting Tsai, Chin-Chu Chen, \& Kwong-Chung Tung

\section{Issue 5}

Immunochemical Similarities in Polysaccharide Components of the Royal Sun CulinaryMedicinal Mushroom, Agaricus brasiliensis (Agaricomycetes), and Clinically Isolated Candida spp.

Hiroaki Tanaka, Chiho Yanai, Ken-ichi Ishibashi, Daisuke Yamanaka, Yoshiyuki Adachi, Koji Araki, Shota Yonetani, Hiroaki Ohnishi, Takao Shinohara, \& Naohito Ohno

Healing Effect of Ethanolic Extracts from Mycelia of the Golden Oyster Mushroom, Pleurotus citrinopileatus (Agaricomycetes), with High Ergothioneine Content in UVB-Irradiated Human Skin Fibroblasts Shin-Yi Lin, Shu-Yao Tsai, \& Jeng-Leun Mau

Lingzhi or Reishi Medicinal Mushroom, Ganoderma Iucidum (Agaricomycetes), Mycelium Aqueous Extract Modulates High-Altitude-Induced Stress Anuja Bhardwaj, Priyanka Sharma, Jigni Mishra, \& Kshipra Misra

Characterization of Exopolysaccharide Produced by Phellinus vaninii (Agaricomycetes) and Antioxidant Potential for Meat Batter Junguang Li, Xuewei Jia, Yanchao Yao, \& Yanhong Bai 
Anti-Inflammatory and Antimicrobial Activities of Aqueous Extracts of Wild Mushrooms from Japan Sayumi Yamada, Mai Tanaka, Rina Miura, Chiaki Takeuchi, Zhihao Tu, Dagula Hu, Keita Matsuoka, Ryota Sugawara, Toshihiro Hoshiba, \& Akihiro Yamaguchi

Medicinal Mushrooms in Complex Treatment of Human Immunodeficiency Virus Infection Tamara V. Teplyakova \& Natalya Gashnikova

Taking Ingredients as an Entry Point to Explore the Relationship between the Shaggy Ink Cap Medicinal Mushroom, Coprinus comatus (Agaricomycetes), and Diabetes Mellitus (Review) Hui Cao, Shiyuan Wang, Xiaowei Cui, Hong Guo, Xiaozhi Xi, Fangxue Xu, Yujuan Li, Mengmeng Zheng, \& Chunchao Han

Combined Administration of the White Button Mushroom Agaricus bisporus (Agaricomycetes) and Lactobacillus casei Modulated Immune-Related Gene Expression and Mucosal and Serum Immune Parameters in a Goldfish (Carassius auratus) Model Roghieh Safari, Seyed Hossein Hoseinifar, Shabnam Nejadmoghaddam, \& Maryam Dadar

Green Mold Caused by Trichoderma atroviride on the Lingzhi Medicinal Mushroom, Ganoderma lingzhi (Agaricomycetes)

Yinhui Yan, Chunlan Zhang, Odeshnee Moodley, Lei Zhang, \& Jize Xu

\section{Issue 6}

A Review on Nutritional and Biological Potential of Earthstar Mushrooms, Species of Genus Astraeus (Agaricomycetes) Pankaj Singh, Vinay K. Varshney, K. Jayaram Kumar, \& Sanjay Singh

Analysis of the Influence of Substrate Formulations on the Bioactive Chemical Profile of Lingzhi or Reishi Medicinal Mushroom, Ganoderma lucidum (Agaricomycetes) by Conventional and Chemometrics Methods Maximiliano A. Bidegain, Pablo D. Postemsky, Olga Pieroni, \& María Amelia Cubitto

Investigations on Antioxidative Potential of Poroid Medicinal Mushroom Porodaedalea pini (Agaricomycetes) Navpreet Kaur, Niharika Kaushal, Minni Singh, Avneet Pal Singh, \& Gurpaul Singh Dhingra

Antioxidant and Anti-Aging Activities of Ethyl Acetate Extract of the Coral Tooth Mushroom, Hericium coralloides (Agaricomycetes) Jinxiu Zhang, Jing Zhang, Liqiang Zhao, Xiaoxi Shui, Li-an Wang, \& Yuefeng Wu

Antioxidant, Anticancer, Antimicrobial, and Antibiofilm Properties of Culinary-Medicinal Fairy Ring Mushroom, Marasmius oreades (Agaricomycetes) Naznoosh Shomali, Okan Onar, Basar Karaca, Nergiz Demirtas, Arzu Coleri Cihan, Ilgaz Akata, \& Ozlem Yildirim

Chaga Medicinal Mushroom, Inonotus obliquus (Agaricomycetes) Polysaccharides Suppress Tacrine-Induced Apoptosis by Reactive Oxygen Species-Scavenging and Mitochondrial Pathway in HepG2 Cells

Zhiguo Li, Junjie Mei, Liping Jiang, Chengyan Geng, Qiujuan Li, Xiaofeng Yao, \& Jun Cao

Increased Inhibition Effect of Antrodin C from the Stout Camphor Medicinal Mushroom, Taiwanofungus camphoratus (Agaricomycetes), on A549 through Crosstalk between Apoptosis and Autophagy Wenhan Wang, Hairui Yang, Jing Deng, Lina Zhu, Yan Yang, Zhendong Liu, Jingsong Zhang, Chuanhong Tang, Zhong Zhang, Haining Zhuang, Henan Zhang, \& Wei Jia

A Comparison of the Physical, Chemical and Structural Properties of Wild and Commercial Strains of the Button Mushroom, Agaricus bisporus (Agaricomycetes) Muhammad Idrees, Sanabil Yaqoob, Sossah Frederick Leo, Asif Ali Khan, Lei Sun, Yang Yang, Dan Li, Yongping $\mathrm{Fu}, \& \mathrm{Yu} \mathrm{Li}$

In Memory of RNDr Václav Šašek CSc (1937-2019) 


\section{Issue 7}

Antiproliferative, Immunostimulatory and Anti-Inflammatory Activities of Extracts Derived from Mushrooms Collected in Haida Gwaii, British Columbia (Canada)

Gurbind S. Deo, Jatinder Khatra, Sukhpreet Buttar, Wai Ming Li, Linda E. Tackaberry,

Hugues B. Massicotte, Keith N. Egger, Kerry Reimer, \& Chow H. Lee

The Inhibitory Properties of Ethanol Extracts of Some Culinary-Medicinal Mushrooms on the Secretion of Interleukin-8 and Vascular Endothelial Growth Factor by PC3 Cancer Cells Christine French, Chandra LC, Stephen Clarke, Edralin A. Lucas, Brenda J. Smith, Stanley Lightfoot, \& Solo R. Kuvibidila

Radical Scavenging and Antiproliferative Effects of Cordycepin-Rich Ethanol Extract from Brown Rice-Cultivated Cordyceps militaris (Ascomycetes) Mycelium on Breast Cancer Cell Lines Han-Chiang Wu, Shou-Tung Chen, Jui-Chih Chang, Ting-Yung Hsu, Chi-Chia Cheng, Huei-Shin Chang, Chin-San Liu, Yao-Chung Wu, \& Zeng-Chin Liang

Comparative Study on the Effect of Aqueous and Ethanolic Mycelial Extracts from Polycephalomyces nipponicus (Ascomycetes) against Human Breast Cancer MCF-7 Cells Benjaporn Buranrat, Kusavadee Sangdee, \& Aphidech Sangdee

Wound Healing Activity of a Skin Substitute from Residues of Culinary-Medicinal Winter Mushroom Flammulina velutipes (Agaricomycetes) Cultivation Hui Xu, Liangyun Liu, Chongjiang Cao, Weisheng Lu, Zhenhan Zhu, Zijun Guo, Min Li, Xing Wang, Dechun Huang, Suilou Wang, \& Shujie Cheng

HS-SPME/GC-MS Assisted Analysis of Volatile Constituents in Different Strains of Shiitake Culinary-Medicinal Mushroom, Lentinus edodes (Agaricomycetes) Lívia Martinez Abreu Soares Costa, Maiara Andrade de Carvalho Sousa, Thiago Pereira Souza, Whasley Ferreira Duarte, Diego Cunha Zied, Danny Lee Rinker, \& Eustáquio Souza Dias

Inhibitory Effect of Five Ganoderma Species (Agaricomycetes) against Key Digestive Enzymes Related to Type 2 Diabetes Mellitus Shao-Dan Chen, Tian-Qiao Yong, Yi-fan Zhang, Hui-Ping Hu, \& Yi-Zhen Xie

Characterization of Anti-Salmonella typhi Compounds from Medicinal Mushroom Extracts from Zimbabwe Tsungai Reid, Chenjerayi Kashangura, Catherine Chidewe, Mudadi A. Benhura, Babill Stray-Pedersen, \& Takafira Mduluza

Mosquitocidal Activity of the Pale Brittle Stem Mushroom, Psathyrella candolleana (Agaricomycetes), against Three Vector Mosquitoes Subramaniyan Sivanandhan, Pathalam Ganesan, Rajendren Host Antony David, Michael Gabriel Paulraj, \& Savarimuthu Ignacimuthu

\section{Issue 8}

Engineering Aspects in Production of Various Medicinal Mushrooms Biomass in Submerged Bioreactors Marin Berovic \& Bojana Boh Podgornik

Bioactivity Guided Isolation of Acetylcholinesterase Inhibitor from Ganoderma mediosinense (Agaricomycetes) Arshdeep Kaur, Kudrat Randhawa, Varinder Singh, \& Richa Shri

Immunomodulating Effects Exerted by Glucans Extracted from the King Oyster Culinary-Medicinal Mushroom Pleurotus eryngii (Agaricomycetes) Grown in Substrates Containing Various Concentrations of Olive Mill Waste Vaclav Vetvicka, Ofer Gover, Hilla Hayby, Ofer Danay, Nirit Ezov, Yitzhak Hadar, \& Betty Schwartz

Profiles of Little-Known Medicinal Polypores: Haploporus odorus (Agaricomycetes) Ivan V. Zmitrovich, Stanislav P. Arefyev, Margarita A. Bondartseva, Nina V. Belova, Yulia R. Khimich, Ludmila G. Isaeva, Vladimir I. Kapitonov, Vyacheslav A. Vlasenko, Sergey V. Volobuev, Oleg N. Ezhov, \& Solomon P. Wasser 
The Immunomodulatory Activity of Polysaccharides from the Medicinal Mushroom Amauroderma rude (Agaricomycetes) Is Mediated via the iNOS and PLA2-AA Pathways Honghui Pan, Xiaojie Zhao, Suwei Lei, Chao Cai, Yizhen Xie, \& Xiaobing Yang

Assessment of Gastroprotective Effect of the Chaga Medicinal Mushroom, Inonotus obliquus (Agaricomycetes), Against the Gastric Mucosal Ulceration Induced by Ethanol in Experimental Rats Xin Xin, Jing Qu, Vishnu Priya Veeraraghavan, Surapaneni Krishna Mohan, \& Kebin Gu

Secondary Metabolites and Antioxidant Capacity of the Tunisian Lichen Diploschistes ocellatus (Ascomycota) Mohamed Mendili, Marwa Bannour, Maria Eduarda M. Araújo, Samira Aschi-Smiti, Mark R.D. Seaward, \& Ayda Khadhri

Antioxidative and Immunomodulatory Activities of the Exopolysaccharides from Submerged Culture of Hen of the Woods or Maitake Culinary-Medicinal Mushroom, Grifola frondosa (Agaricomycetes) by Addition of Rhizoma gastrodiae Extract and Its Main Components Zong-Qi Zhang, Li-Ping Liu, Lu Lei, Chuan-Nan Wang, Qing-Li Tang, \& Tian-Xiang Wu

\section{Issue 9}

Review of Bioactive Molecules Production, Biomass and Basidiomata of Shiitake Culinary-Medicinal Mushrooms, Lentinus edodes (Agaricomycetes) Rigoberto Gaitán-Hernández, Damián López-Peña, Martín Esqueda, \& Aldo Gutiérrez

Comprehensive Evaluation of Shiitake Culinary-Medicinal Mushroom Strains (Lentinus edodes, Agaricomycetes) Based on Polysaccharides Content and Agronomic Traits Xue-li Zheng, Wen-bing Gong, Chuang Li, Lin Zhang, Yin-bing Bian, Hoi-shan Kwan, Man-kit Cheung, \& Yang Xiao

The Antioxidant Properties, Tyrosinase and $\alpha$-Glucosidase Inhibitory Activities of Phenolic Compounds in Different Extracts from the Golden Oyster Mushroom, Pleurotus citrinopileatus (Agaricomycetes) Chaomin Yin, Xiuzhi Fan, Chunyou Liu, Zhe Fan, Defang Shi, Fen Yao, Wei Cheng, \& Hong Gao

Review of Advances in Bioactive Low-Molecular-Weight Compounds, Extracts, and Biology of Phellinus sensu lato Mushrooms (Agaricomycetes) from 2011 to 2017 Yating Dong, Haile Ma, Cunshan Zhou, Golly K. Moses, Xiaofei Ye, Henan Zhang, Ling Sun, \& Mohammed A.Y. Abdualrahman

The Effects of Ethanol Concentration during Isolation and Purity on the In Vitro Antioxidant Properties of Polysaccharides from the Winter Culinary-Medicinal Mushroom, Flammulina velutipes (Agaricomycetes) Stembase Zhixin Guo, Li Zhao, Dong Zhen, Mahfuz Shad, Hanyu Xu, Hui Song, \& Dongming Zhang

The DnaJ Gene Family in Shiitake Culinary-Medicinal Mushroom, Lentinus edodes (Agaricomycetes): Comprehensive Identification, Characterization, and Expression Profiles under Different Conditions Gangzheng Wang, Yi Luo, Wang Chen, Yuhua Gong, Yan Zhou, \& Yinbing Bian

Efficient Transformation of the White Jelly Mushroom Tremella fuciformis (Tremellomycetes) and Its Companion Fungus Annulohypoxylon stygium (Ascomycetes) Mediated by Agrobacterium tumefaciens Dongmei Liu, Hanyu Zhu, Liesheng Zheng, Liguo Chen, \& Aimin Ma

Identification, Optimization of Culture Conditions, and Bioactive Potential of Chinese Caterpillar Mushroom Ophiocordyceps sinensis (Ascomycetes) Mycelium Isolated from Fruiting Body Vikas Kaushik, Aditi Arya, Anil Sindhu, \& Ajay Singh

\section{Issue 10}

Phylogenetic Comparison between Italian and Worldwide Hericium Species (Agaricomycetes) Valentina Cesaroni, Maura Brusoni, Carlo Maria Cusaro, Carolina Girometta, Claudia Perini, Anna Maria Picco, Paola Rossi, Elena Salerni, \& Elena Savino 
Exogenous Salicylic Acid (SA) Promotes the Accumulation of Biomass and Flavonoid Content in Phellinus igniarius (Agaricomycetes)

Liang Shi, Yingru Tan, Zehua Sun, Ang Ren, Jing Zhu, \& Mingwen Zhao

Composition of Mycelia and Basidiomata of the Culinary-Medicinal Golden Oyster Mushroom, Pleurotus citrinopileatus (Agaricomycetes) by Nuclear Magnetic Resonance Spectroscopy Shin-Yi Lin, Carolyn M. Slupsky, Darya O. Mishchuk, \& Jeng-Leun Mau

Evaluation of Bioactivities, Phenolic and Metal Content of Ten Wild Edible Mushrooms from Western Black Sea Region of Turkey Tevfik Ozen, Demet Klzll, Semiha Yenigun, Hasan Cesur, \& Ibrahim Turkekul

Isolation, Anti-Inflammatory Activity and Physicochemical Properties of Bioactive Polysaccharides from Fruiting Bodies of Cultivated Cordyceps cicadae (Ascomycetes) Chun-Hsien Yang, Chun-Han Su, Shou-Chou Liu, \& Lean-Teik Ng

Effects of Extraction Conditions on Crude Polysaccharides and Antioxidant Activities of the Lion's Mane Medicinal Mushroom, Hericium erinaceus (Agaricomycetes)

Shengjuan Jiang, Shanshan Liu, \& Meisong Qin

The Antioxidative, Anti-Inflammatory and Liver-Protective Effects of Mycelia Selenium Polysaccharides from the Deep Root Mushroom, Oudemansiella radicata (Agaricomycetes) Hui Liu, Qihang Yang, Zheng Gao, Yongfa Zhu, Jianjun Zhang, \& Le Jia

Hypoglycemic Effect of the Degraded Polysaccharides from the Wood Ear Medicinal Mushroom Auricularia auricula-judae (Agaricomycetes) Meng Shen, Zhiyu Fang, Yutao Chen, Yidan Chen, Bin Xiao, Li Guo, Yaoyao Xu, Ge Wang, Weimin Wang, \& Yongjun Zhang

The Effects of Lentinan on the Expression Patterns of $\beta$-Catenin, Bcl-2 and Bax in Mouse Bone Marrow Cells Are Associated with Enhancing Dectin-1 Yong Zhou, Xia-Liang Chen, \& Zhen-Yue Ye

\section{Issue 11}

Characterization of Biological Activities of Methanol Extract of Fuscoporia torulosa (Basidiomycetes) from Italy Stefano Covino, Eleonora D’Ellena, Bruno Tirillini, Giancarlo Angeles, Andrea Arcangeli, Giancarlo Bistocchi, Roberto Venanzoni, \& Paola Angelini

A Polysaccharide Extract from Maitake Culinary-Medicinal Mushroom, Grifola frondosa (Agaricomycetes) Ameliorates Learning and Memory Function in Aluminum Chloride-Induced Amnesia in Mice Linlin Fan, Lingling Chen, Zhi Liang, Hongkun Bao, Dan Wang, Yilong Dong, Shangyong Zheng, Chunjie Xiao, Jing Du, \& Hongliang Li

Chemical Profile, In Vitro Enzyme Inhibitory, and Antioxidant Properties of Stereum Species (Agaricomycetes) from Turkey Fatih Çayan, Gülsen Tel-Çayan, Ebru Deveci, Mehmet Öztürk, \& Mehmet Emin Duru

Effect of Aging on Culture and Cultivation of the Culinary-Medicinal Mushrooms Morchella importuna and M. sextelata (Ascomycetes) Peixin He, Miao Yu, Yingli Cai, Wei Liu, Wensheng Wang, Shaohua Wang, \& Jing Li

Medicinal, Nutritional, and Cosmetic Values of Macrofungi Distributed in Mazandaran Province of Northern Iran (Review) Susanna M. Badalyan \& Ali Borhani

Effect of Culture Time on the Bioactive Components in the Fruit Bodies of Caterpillar Mushroom, Cordyceps militaris CM-H0810 (Ascomycetes) Lina Zhu, Hongxia Zhang, Yangfang Liu, Jingsong Zhang, Xinhua Gao, \& Qingjiu Tang 
Evaluation of Antiarthritic Effect of Culinary-Medicinal Oyster Mushroom Pleurotus ostreatus cv. Florida (Agaricomycetes) on Complete Freund's Adjuvant Induced Arthritis in Rats Ayushi Chourasia, Ankita Tiwari, Aditya Ganeshpurkar, Anupam Jaiswal, Abhishek Shrivastava, \& Nazneen Dubey

Therapeutic Effect and Potential Mechanisms of Lion's Mane Medicinal Mushroom, Hericium erinaceus (Agaricomycetes), Mycelia in Submerged Culture on Ethanol-Induced Chronic Gastric Injury Xiangjunzhi Mao, Zhen-Ming Lu, Ting-Ting Gong, Kang-Le Wang, Yan Geng, Hong-Yu Xu, Guo-Hua Xu, Jin-Song Shi, \& Zheng-Hong Xu

\section{Issue 12}

Activation of the AKT/FOXO3a Signaling Pathway during Breast Cancer Inhibition In Vivo and In Vitro by Amauroderma rude (Agaricomycetes) Honghui Pan, Suwei Lei, Xiaojie Zhao, Yizhen Xie, \& Xiaobing Yang

Supplementation of Lingzhi or Reishi Medicinal Mushroom, Ganoderma lucidum (Agaricomycetes) Extract Enhanced the Medicinal Values and Prebiotic Index of Hungarian Acacia Honey Attila Kiss, Iman Mirmazloum, Zoltán Naár, \& Erzsébet Némedi

Increasing of the Contain of Carotenoids in Caterpillar Mushroom, Cordyceps militaris (Ascomycetes) by Using the Fungal Elicitors Cultivation Hongbiao Tang, Zhiwei Ye, Cong Liu, Liqiong Guo, Junfang Lin, Hua Wang, Fan Yun, \& Lingzhi Kang

Identification and In Silico Analysis of Lectins in Gray Oyster Culinary-Medicinal Mushroom Pleurotus ostreatus (Agaricomycetes) Based on the Transcriptomes Danyun Xu, Jing Lu, Yuanyuan Wang, Aafaque Ahmed Keerio, Liesheng Zheng, Liguo Chen, \& Aimin Ma

Employment of ARTP to Generate Phellinus baumii (Agaricomycetes) Strain with High Flavonoids Production and Validation by Liquid Fermentation

Henan Zhang, Fuchun Jiang, Dehui Qu, Wenhan Wang, Yating Dong, Jingsong Zhang, Di Wu, \& Yan Yang

The Reliability of DNA Sequences in Public Databases Belonging to the Most Economically Important Shiitake Culinary-Medicinal Mushroom Lentinus edodes (Agaricomycetes) in Asia Rui-Heng Yang, Ying-Ying Wu, Li-Hua Tang, Chuan-Hua Li, Jun-Jun Shang, Yan Li, Ying Song, Wei-Hua Huang, Xiang-Sheng Tao, Qi Tan, \& Da-Peng Bao

The Brown Roll-Rim Mushroom, Paxillus involutus (Agaricomycetes), as a Promising Biomedical Research Resource Ivan V. Zmitrovich, Nina V. Belova, Nadezhda V. Psurtseva, \& Solomon P. Wasser

Index, Volume 21, 2019 


\section{INTERNATIONAL JOURNAL OF MEDICINAL MUSHROOMS}

Author Index, VOLUME 21, 2019

Page Range of Issues - Issue 1: 1-103; Issue 2: 105-206; Issue 3: 207-309; Issue 4: 311-411; Issue 5: 413-521; Issue 6: 523-628; Issue 7: 629-734; Issue 8: 735-839; Issue 9: 841-942; Issue 10: 943-1050; Issue 11: 1051-1150; Issue 12: 1151-1247

Abdualrahman, M.A.Y., 875

Abdul-Samad, J.L., 131

Actis, L.A., 131

Adachi, Y., 413

Agrawal, M., 29

Akata, I., 571

Akubugwo, E.I., 193

Alabiso, M., 89

Angeles, G., 1051

Angelini, P., 1051

Anike, F.N., 89

Anwar, H., 79

Araki, K., 413

Araújo, M.E.M., 817

Arcangeli, A., 1051

Arefyev, S.P., 783

Arya, A., 931

Aschi-Smiti, S., 817

Awasthi, R., 29

Ayinde, B.A., 143

Badalyan, S.M., 291, 1099

Bai, Y., 459

Balandaykin, M.E., 105

Baldissera, B.L., 169

Bamigboye, C.O., 311

Bannour, M., 817

Bansal, A., 155

Bao, D.-P., 1223

Bao, H., 1065

Becker, T.L., 131

Belova, N.V., 105, 783, 1241

Benhura, M.A., 713

Berovic, M., 253, 735

Bharat Dalvi, Y.B., 367

Bhardwaj, A., 443

Bian, Y., 909

Bian, Y.-b., 851

Bidegain, M.A., 537

Bistocchi, G., 1051

Bonanno, A., 89

Bondartseva, M.A., 105, 783

Borhani, A., 1099

Brezáni, A., 215

Brusoni, M., 943

Buranrat, B., 671

Burton, M., 311

Buttar, S., 629
Cai, C., 793

Cai, Y., 1089

Cao, C., 683

Cao, H., 493

Cao, J., 583

Çayan, F., 1075

Cesaroni, V., 943

Cesur, H., 979

Chandra L.C., 645

Chandra, R., 181

Chang, H.-S., 657

Chang, J.-C., 657

Chen, B., 381

Chen, C.-C., 401

Chen, J., 381

Chen, L., 921, 1065, 1193

Chen, S.-D., 703

Chen, S.-T., 657

Chen, W., 909

Chen, W.-P., 401

Chen, X.-L., 1043

Chen, Y., 1033

Cheng, C.-C., 657

Cheng, S., 683

Cheng, W., 865

Cheung, M.-k., 851

Chidewe, C., 713

Chinnaboina, G.K., 323

Chourasia, A., 1123

Cihan, A.C., 571

Ćilerdžić, J., 275

Clarke, S., 645

Costa, L.M.A.S., 693

Covino, S., 1051

Cubitto, M.A., 537

Cui, X., 493

Cusaro, C.M., 943

D’Ellena, E., 1051

Dadar, M., 503

Dahiya, R., 29

Dames, J.F., 311

Danay, O., 765

Danyun Xu, D., 1193

David, R.H.A., 725

de Carvalho Sousa, M.A., 693

de Franceschi de Angelis, D., 169
Demirtas, N., 571

Deng, J., 595

Deng, W.-Q., 381

Deo, G.S., 629

Deveci, E., 1075

Dhingra, G.S.,549

Di Grigoli, A., 89

Di Miceli, G., 89

Dias, E.S., 693

Donatini, B., 267

Dong, C., 59

Dong, Y., 875, 1065, 1207

Du, J., 1065

Dua, K., 29

Duarte, W.D., 693

Dubey, N., 1123

Duru, M.E., 1075

Egger, K.N., 629

Elisashvili, V., 1115

Emmanuel, O., 193

Esqueda, M., 841

Ezhov, O.N., 783

Ezov, N., 765

Fan, L., 1065

Fan, X., 865

Fan, Z., 865

Fang, Z., 1033

Feng, N., 207

French, C., 645

Fu, Y., 611

Gabriel, J., 627

Gaitán-Hernández, R., 841

Galić, M., 275

Ganesan, K., 237

Ganesan, P., 725

Ganeshpurkar, A., 1123

Gangzheng Wang, G., 909

Gao, H., 865

Gao, X., 1107

Gao, Z., 1019

Gargano, M.L., 89, 225

Gashnikova, N., 487

Gdula-Argasińska, J., 343

Geng, C., 583

Geng, Y., 1137

Girometta, C., 943

Gong, T.-T., 1137 
Gong, W.-b., 851

Gong, Y., 909

Gover, O., 765

Grodzinskaya, A.A., 71

Gu, K., 805

Guo, H., 493

Guo, L., 47, 1033, 1181

Guo, L.-Q., 353

Guo, X.-Y., 353

Guo, Z., 683, 895

Gupta, G., 29, 323

Gutiérrez, A., 841

Hadar, Y., 765

Han, C., 493

Han, L., 207

Han, W., 207

Hassan, F., 131

Hayby, H., 765

He, H., 381

He, P., 1089

Honchar, H.Yu., 71

Hoseinifar, S.H., 503

Hoshiba, T., 469

Hsu, T.-Y., 657

Hu, D., 469

Hu, H.-P., 703

Huang, D., 683

Huang, W., 381

Huang, W.-H., 1223

Hussain, G., 79

Ibe, C., 193

Idrees, M., 611

Ignacimuthu, S., 725

Isaeva, L.G., 783

Ishibashi, K.-I., 413

Isikhuemhen, O.S., 89

Islam, T., 237

Jablonský, I., 215, 627

Jaiswal, A., 1123

Jia, L., 1019

Jia, W., 595

Jia, X., 459

Jiang, F., 1207

Jiang, L., 583

Jiang, S., 1007

Jiang, X., 301

Kachlishvili, E., 1115

Kała, K., 343

Kang, L., 47, 1181

Kapitonov, V.I., 783

Karaca, B., 571

Kashangura, C., 713

Kaur, A., 755

Kaur, N., 549

Kaushal, N., 549

Kaushik, V., 931

Keerio, A.A., 1193

Kennedy, M.A., 131
Khadhri, A., 817

Khan, A.A., 611

Khan, F., 181

Khatra, J., 629

Khimich, Y.R., 783

Kinstedt, C.M., 131

Kiss, A., 1167

K1z11, D., 979

Kobakhidze, A., 1115

Krakowska, A., 343

Kumar, K.J., 523

Kunca, V., 121

Kuvibidila, S.R., 645

Kwan, H.-s., 851

Lamrood, P.Y., 367

Lateef, A., 311

Le Blaye, I., 267

Lee, C.H., 629

Lee, L.-Y., 401

Lei, L., 825

Lei, S., 793, 1151

Leo, S.F., 611

Li, C., 851

Li, C.-H., 1223

Li, D., 611

Li, F., 331

Li, H., 1065

Li, I.-C., 401

Li, J., 459, 1089

Li, M., 683

Li, Q., 583

Li, S., 381

Li, W.M., 629

Li, Y., 13, 1223

Li, Y., 493, 611

Li, Z., 583

Liang, Z., 1065

Liang, Z.-C., 657

Lightfoot, S., 645

Lin, J., 47, 1181

Lin, J.-F., 353

Lin, S.-Y., 429, 965

Lin, Y., 47, 301

Liu, C., 865, 1181

Liu, C.-S., 657

Liu, D., 921

Liu, G., 59

Liu, H., 1019

Liu, K., 59

Liu, L., 381, 683

Liu, L.-P., 825

Liu, S., 1007

Liu, S.-C., 995

Liu, W., 207, 1089

Liu, X., 323

Liu, Y., 13, 1107

Liu, Z., 595

López-Peña, D., 841
Lou, H.-W., 353

Lu, J., 1193

Lu, W., 683

Lu, Z.-M., 1137

Lucas, E.A., 645

Luo, Y., 909

Lv, H., 381

Ma, А., 921, 1193

Ma, H., 875

Ma, L., 301

Maier, R.J., 1

Maier, S.E., 1

Massicotte, H.B., 629

Matsuoka, K., 469

Mau, J.-L., 429, 965

Mduluza, T., 713

Mei, J., 583

Mendili, M., 817

Michalak-Majewska, M., 393

Mirmazloum, I., 1167

Mishchuk, D.O., 965

Mishra, A., 29, 323

Mishra, J., 155, 443

Misra, K., 155, 443

Miura, R., 469

Mohan, S.K., 805

Moodley, O., 515

Morales, E.M., 169

Moses, G.K., 875

Mustafa, I., 79

Muszyńska, B., 343

Naár, Z., 1167

Nair, C.K.K., 367

Nebesnyi, V.I., 71

Nejadmoghaddam, S., 503

Némedi, E., 1167

Ng, L.-T., 995

Ni, S., 131

Ohnishi, H., 413

Ohno, N., 413

Okomba, N.O., 193

Oloke, J.K., 311

Onar, O., 571

Onyero, O., 193

Opoka, W., 343

Ozen, T., 979

Ozolua, R.I., 143

Öztürk, M., 1075

Pan, H., 793, 1151

Pathak, S., 29

Paulraj, M.G., 725

Pavlík, M., 121

Perini, C., 943

Picco, A.M., 943

Pieroni, O., 537

Podgornik, B.B., 253, 735

Postemsky, P.D., 537

Psurtseva, N.V., 1241 
Qiao, J., 331

Qin, M., 1007

Qu, D., 1207

Qu, J., 805

Radzki, W., 393

Rajput, R., 155

Randhawa, K., 755

Rao, S., 331

Reid, T., 713

Reimer, K., 629

Ren, A., 955

Rinker, D.L., 693

Rossi, P., 943

Rusitashvili, M., 1115

Safari, R., 503

Salerni, E., 943

Samchuk, A.I., 71

Sangdee, A., 671

Sangdee, K., 671

Saporita, P., 225

Savino, E., 943

Schwartz, B., 765

Seaward, M.R.D., 817

Shabbir, M.Z., 79

Shad, M., 895

Shang, J.-J., 1223

Sharma, P., 443

Sharma, R.K., 29, 323

Shen, M., 1033

Shi, D., 865

Shi, J.-S., 1137

Shi, L., 955

Shinohara, T., 413

Shnyreva, A.A., 291

Shnyreva, A.V., 291

Shomali, N., 571

Shri, R., 755

Shrivastava, A., 1123

Shuai, Y., 331

Shui, X., 561

Sindhu, A., 931

Singh, A., 931

Singh, A.P., 549

Singh, K., 155

Singh, M., 323, 549

Singh, P., 523

Singh, S., 523

Singh, V., 755

Sivanandhan, S., 725

Skrzypczak, K., 393

Sławińska, A., 393

Slupsky, C.M., 965

Smith, B.J., 645

Sobowale, M.T., 143

Soderberg, C., 205

Sohail, M.U., 79

Song, H., 895

Song, Y., 1223
Souza, T.P., 693

Stajić, M., 275

Stray-Pedersen, B., 713

Su, C.-H., 995

Su, L., 13

Suchodolski, J.S., 79

Sugawara, R., 469

Sun, L., 611, 875

Sun, Z., 955

Svobodová, K., 215

Tackaberry, L.E., 629

Takeuchi, C., 469

Tan, Q., 1223

Tan, Y., 955

Tanaka, H., 413

Tanaka, M., 469

Tang, C., 595

Tang, H., 1181

Tang, L.-H., 1223

Tang, Q., 37, 1107

Tang, Q.-J., 207

Tang, Q.-L., 825

Tao, X.-S., 1223

Tel-Çayan, G., 1075

Teplyakova T.V., 487

Tirillini, B., 1051

Tiwari, A., 1123

Tlustoš, P., 215

Todaro, M., 89

Tsai, S.-Y., 429

Tsai, Y.-T., 401

Tsokilauri, A., 1115

Tu, Z., 469

Tung, K.-C., 401

Turkekul, I., 979

Ude, V.C., 193

Ugbogu, E.A., 193

Ullah, M.I., 79

Uwaya, D.O., 143

Varghese, R., 367

Varshney, V.K., 523

Veeraraghavan, V.P., 805

Venanzoni, R., 1051

Venturella, G., 89, 225

Vetvicka, V., 765

Vitale, F., 89

Vlasenko, V.A., 783

Volobuev, S.V., 783

Vukojević, J., 275

Wang, A., 381

Wang, C.-N., 825

Wang, D., 13, 1065

Wang, F., 59

Wang, G., 1, 1033

Wang, H., 1181

Wang, J., 381

Wang, K.-L., 1137

Wang, L.-i., 561
Wang, Q., 13

Wang, S., 1089

Wang, S., 37, 493, 683

Wang, W., 595, 1033, 1089, 1207

Wang, X., 683

Wang, Y., 1193

Wasser, S.P., 105, 783, 1241

Wei, T., 47

Wei, Y.-T., 207

Wu, D., 1207

Wu, F., 37

Wu, H.-C., 657

Wu, T.-X., 825

Wu, Y., 561

Wu, Y.-C., 657

Wu, Y.-Y., 1223

$\mathrm{Xi}, \mathrm{X} ., 493$

Xiangjunzhi Mao,

Xiao, B., 1033

Xiao, C., 1065

Xiao, D., 301

Xiao, Y., 851

Xie, Y., 793, 1151

Xie, Y.-Z., 703

Xin, X., 805

Xu, B., 237

$\mathrm{Xu}, \mathrm{D} ., 331$

Xu, F., 493

Xu, G.-H., 1137

Xu, H., 683, 895

Xu, H.-Y., 1137

$\mathrm{Xu}, \mathrm{J} ., 515$

Xu, Y., 1033

Xu, Z.-H., 1137

Yamada, S., 469

Yamaguchi, A., 469

Yamanaka, D., 413

Yan, Y., 515

Yanai, C., 413

Yang, C., 301

Yang, C.-H., 995

Yang, H., 595

Yang, Q., 1019

Yang, R.-H., 1223

Yang, X., 793, 1151

Yang, Y., 595, 611, 1207

Yao, F., 865

Yao, X., 583

Yao, Y., 459

Yaqoob, S., 611

Ye, X., 875

Ye, Z., 47, 1181

Ye, Z.-Y., 1043

Yenigun, S., 979

Yildirim, O., 571

Yin, C., 865

Ying, Z., 301 
Yonetani, S., 413

Yong, T.-Q., 703

Yu, M., 1089

Yun, F., 47, 1181

Zeng, H., 331

Zeng, X., 331

Zhang, C., 515

Zhang, D., 895

Zhang, H., 595, 875, 1107, 1207

Zhang, J., 37, 561, 595, 1019, 1107, 1207

Zhang, J.-S., 207

Zhang, L., 1, 515, 851
Zhang, X., 1

Zhang, X.-C., 353

Zhang, Y., 1033

Zhang, Y.-f., 703

Zhang, Z., 37, 595

Zhang, Z.-Q., 825

Zhao, L., 561, 895

Zhao, M., 955

Zhao, X., 793, 1151

Zhen, D., 895

Zheng, D., 13

Zheng, L., 921, 1193

Zheng, M., 493

Zheng, Q., 47
Zheng, S., 1065

Zheng, X.-l., 851

Zhou, C., 875

Zhou, S., 37

Zhou, Y., 909, 1043

Zhu, H., 921

Zhu, J., 955

Zhu, L., 37, 595, 1107

Zhu, Y., 1019

Zhu, Z., 683

Zhuang, H., 595

Zied, D.C., 693

Zmitrovich, I.V., 105, 783, 1241 


\title{
INTERNATIONAL JOURNAL OF MEDICINAL MUSHROOMS
}

\author{
SUBJECT INDEX, VOLUME 21, 2019
}

Page Range of Issues - Issue 1: 1-103; Issue 2: 105-206; Issue 3: 207-309; Issue 4: 311-411; Issue 5: 413-521; Issue 6: 523-628; Issue 7: 629-734; Issue 8: 735-839; Issue 9: 841-942; Issue 10: 943-1050; Issue 11: 1051-1150; Issue 12: 1151-1247

antibacterial, 155

137Cs, 71

13-week subchronic rodent feeding study, 401

16S rRNA sequencing, 79

2-stage process, 47

5.8S rRNA sequence, 725

8-oxo-guanine, 1

ABTS, 393

ABTS, 561

acacia honey, 1167

accumulation, 71

acetaminophen, 367

acetylcholinesterase inhibition, 275, 755

acid hydrolysates, 1033

adaptogens, 443

adsorption, 169

advanced glycation end

products, 1033

Agaricus bisporus, 13, 181, 503, 611, 645

Agaricus brasiliensis, 169, 413

Agaricus campestris, 181

AgHWE, 413

Agrobacterium tumefaciens-

mediated transformation, 921

air lift bioreactors, 253, 735

alternative growth substrates, 215

alternative substrates, 841

altitude, 121

aluminum chloride, 1065

Amauroderma rude, 793, 1151

amino acids, 493

amnesia, 1065

angiogenesis, 645

Annulohypoxylon stygium, 921

antibiofilm activity, 571

antibiotic activity, 131

antibiotic resistance, 131

antibiotic, 131

anticancer activity, 671

anticancer, 571

anticholinesterase activity, 1075

antiepileptic activity, 29

antihyperlipidemia, 193 anti-inflammatory, 629, 995, 1019

antimicrobial activity, 979

antimicrobial compounds, 713

antimicrobial, 571, 1051

antioxidant activity, 459, 549, 825, 865, 979, 1007, 1075,

1167

antioxidant component, 353

antioxidant enzymes, 367

antioxidant potential, 237

antioxidants, 143, 155, 207,

393, 571, 583, 755, 895

antioxidation, 657

antioxidative capacity, 275

antioxidative, 1019

antiproliferation, 657

antiproliferative, 629

antitumor activity, 331

antitumor, 1151

apoptosis, 583, 595, 657

arthritis, 1123

artificial neural network, 311

ARTP, 1207

Ascomycetes, 225

Aspergillus niger, 181

Astraeus spp., 523

Auricularia auricula-judae

polysaccharide, 1033

Auricularia polytricha, 29, 323

autophagy, 595

Basidiomycetes, 193, 225

batch, 253, 735

Bax, 1043

Bcl-2, 1043

bioactive compounds, 875

bioactive ingredients, 331

bioactive metabolites, 841

bioactive potential, 931

bioactivity, 817

biodistribution, 443

bioindication, 71

biological activities, 523

biological activity, 487, 875

bioprospecting, 181

biotechnological potential, 1099

breast cancer, 657
CaCo-2 cells, 343

cadmium, 169

CAHWE, 413

Canada, 629

cancer hallmarks, 105

cancer therapy, 1241

Candida albicans, 413

Candida spp., 413

Cantharellus heinemannianus, 713

Cantharellus miomboensis, 713

Cantharellus symoensii,713

carotenoids accumulation, 1181

carotenoids, 47

catalepsy, 323

CAWS, 413

cell adhesion, 1

cell clone, 595

cell cycle, 595

cell migration, 595

cellulase, 1115

Cephalosporium sinensis, 331

chaga mushroom, 805

chamonixin, 1241

cheese oxidative stability, 89

chelating power, 817

chemical compositions, 331

chemical constituents, 523

chromium, 169

Cinnamomum verum, 267

cis elements, 909

complex therapy of AIDS, 487

composition profile, 965

comprehensive evaluation, 851

concanavalin A, 413

copper, 537

Coprinus comatus, 493

Cordyceps cicadae, 331, 995

Cordyceps militaris, 47, 331, 657, 735, 1107, 1181

Cortinarius balteatocumatilis, 469

Crohn disease, 267

CSBG, 413

cultural characteristics, 1241

culture conditions, 931

dectin-1, 1043 
degree of purification, 895

D-galactose-induced aged

mouse model, 561

diabetes mellitus, 493

dietary fibers, 493

Diploschistes ocellatus, 817

dissolution rate, 37

distribution, 783

DnaJ, 909

DPPH, 561, 1051

drimane sesquiterpenoids, 783

dyslipidemia, 79

edible and medicinal

mushrooms, 121, 237, 301,

611

edible, 1099

enhanced green fluorescent

protein, 921

entomopathogenic fungus, 671

ergothioneine, 429

erinacine A-enriched Hericium

erinaceus mycelia, 401

Escherichia coli, 181, 469

estrogen signals, 381

ewes, 89

exopolysaccharide, 459

expression profile, 909, 1193

extraction into artificial

digestive system, 343

extraction, 1007

extracts, 875

fatty acids, 89, 1075

fed-batch cultivation, 253, 735

fermented conifer sawdust, 215

field cultivation, 1089

Flammulina velutipes residues, 683

Flammulina velutipes stembase, 895

flavonoids, 1207

Fomitopsis pinicola, 291

food function, 469

Fourier transform-infrared

spectroscopy, 611

FOXO3a, 1151

FRAP, 393

freeze drying, 393

fruiting body, 931

fungal elicitors, 1181

fungi, 995

fungus myceliated grains, 89

Fuscoporia torulosa, 1051

gallic acid, 755

Ganoderma lingzhi, 515

Ganoderma lucidum extract, 143

Ganoderma lucidum, 215, 253, 353, 443, 537, 1167
Ganoderma mediosinense, 755

Ganoderma, 703

gas chromatography-mass

spectrometry, 207, 693

gastric injury, 1137

gastric ulcer, 805

gastroprotective, 805

gene expression, 503

genetic variability, 291

glucans, 393, 765

goldfish, 503

grain, 353

grey relational analysis, 851

Grifola frondosa, 735, 825, 1065

growth receptors, 1241

gynecological cancers, 225

Haida gwaii, 629

haloperidol, 323

haploporic acid A, 783

Haploporus odorus, 783

Haploporus, 783

HcEAE, 561

headspace sampling coupled

with gas chromatography-mass

spectrometry (HS/GC-MS), 693

health risk, 169

heavy metal contamination, 169

Helicobacter pylori, 1

hematopoiesis, 193

hemolysis, 1241

hepatoprotection, 311, 367

hepatotoxicity, 193

Hericium coralloides, 561

Hericium erinaceus, 1, 735, 1007, 1137

Hericium, 943

hormone replacement therapy, 381

hot-air drying, 393

human immunodeficiency virus, 225

human papillomavirus, 225

human skin fibroblasts, 429

hydroxyl, 561

hypoglycemic effect, 493, 1033

hypoxia, 443

identification, 515

Imleria badia, 343

immune tolerance, 105

immunocomplex-mediated

hypersensitivity of type III, 1241

immunodeficiency virus, 487 immunomodulatory activity, 793, 825 immunomodulatory properties, 487

immunomodulatory, 13

immunostimulatory, 629

immunotherapy, 105

in silico analysis, 1193

in silico, 1223

in vitro digestion model, 1167

India, 181

indole compounds, 343

inducers, 47

inflammation, 765, 1123

inflammatory bowel disease, 765

infrared spectroscopy,537

ingredients, 493

Inonotus obliquus, 583, 805

iNOS, 793

interleukin-8 (IL-8), 645

interleukin-8, 1

internal transcribed spacer, 931, 943, 1223

intestinal parasite control, 89

involutin, 1241

Isaria cicadae, 59

isolation, 895

isoniazid, 29

iTRAQ, 1137

ITS1-ITS4, 1051

keratin, 1137

laccase, 1115

Lactobacillus casei, 503

Laetiporus sulphureus, 275, 267

larvicidal activity, 725

laser light scattering, 459

lead toxicity, 143

lead, 169

lectin, 1193

lentinan, 1043

Lentinus edodes, 37, 79, 645,

693, 841, 851, 909, 1043, 1223

lichens, 817

light, 59, 301

lipid levels, 143

liver enzymes, 143

liver protection, 1019

low-molecular-weight

compounds, 875

lucidenic acid M, 713

M. sextelata, 1089

macrofungi, 1099

maitake, 1065

manganese peroxidase, 1115

mannitol, 1107

Marasmius oreades, 571

mass spectrometry, 713

maximal electroshock, 29

mean annual temperature, 121 
medicinal and edible

mushrooms, 979

medicinal mushrooms, 1,13 , 29, 37, 47, 59, 71, 79, 89, 105 $131,143,155,169,181,193$, 207, 215, 225, 253, 267, 275, 291, 311, 323, 331, 343, 353, 367, 381, 393, 401, 413, 429, 443, 459, 469, 487, 493, 503, 515, 523, 537, 549, 561, 571, 583, 595, 629, 645, 657, 671, 683, 693, 703, 713, 725, 735, 755, 765, 783, 793, 805, 825, 841, 851, 865, 875, 895, 909, 921, 931, 943, 955, 965, 995, 1007, 1019, 1033, 1043, 1051, 1065, 1075, 1089, 1107, 1115, 1123, 1137, 1151, 1167, 1181, 1193, 1207, 1223, 1241

medicinal polypores, 783

medicinal value of lichens and fungi, 817

medicinal, 1099

metabolic disorders, 79

metal, 979

methyl-hispolon, 381

microbiome, 79

milk, 89

mineral elements, 71

minimum inhibitory

concentration, 131

mitochondrial pathway, 583

molecular code, 291

mono- and dikaryotic

basidiomycetes, 1115

Morchella importuna, 1089

morel, 1089

morphological characteristics, 611

morphological identification,

943

morphology and rheology, 735

morphology, 783

mouse colonization, 1

mucosal immunity, 503

mushroom cultivation, 215

mushroom production, 169

mutation, 1207

mycelia, 1137

mycelial growth, 1089

mycelium from in vitro culture, 343

mycochemicals, 237

N6-(2-hydroxyethyl)-adenosine, 59

natural peptides, 155

neurodegenerative disease, 401

neuroprotection, 1065 new disease, 515

new subtype A with HIV-1

CRF63_02A1, 487

normalization, 301

Northern Iran, 1099

nuclear magnetic resonance, 965

nuclear ribosomal DNA, 943

nucleoside, 59, 1107

nutritional value, 523

olive oil, 537

Ophiocordyceps sinensis, 155 , 931

optimization, 311

Origanum vul-gare, 267

Oudemansiella radicata, 1019

oxidative stress, 323, 1089

oxygen transfer, 735

Paecilomyces hepiali, 331

p-AKT, 1151

paw volume, 1123

Paxillus involutus complex, 1241

Paxillus syndrome, 1241

PC3 cells, 645

Phellinus baumii, 1207

Phellinus caryophylli, 367

Phellinus igniarius, 955

Phellinus lonicerinus, 381

Phellinus sensu lato, 875

Phellinus vaninii, 459

phenolic and flavonoid compounds, 1051

phenolic composition, 571

phenolic compounds, 865, 1075

phenolic content, 817

phenolics, 979

phenytoin, 29

phosphotransferase, 921

p-hydroxylbenzaldehyde, 825

phylogenetic analysis, 291

phylogenetic tree, 725

phylogeny, 943

phytoestrogen, 381

PLA2-AA, 793

Pleurotus cictrinopileatus, 965

Pleurotus citrinopileatus, 429, 865

Pleurotus eryngii, 765

Pleurotus ostreatus cv. Florida, 1123

Pleurotus ostreatus, 275, 1193

Pleurotus spp., 215

Pleurotus tuberregium, 311

Polycephalomyces nipponicus, 671

polyglobin N, 413

Polyporus umbellatus, 121 polysaccharide content, 851

polysaccharides, $13,37,393$,

493, 895, 995, 1065, 1107

Porodaedalea pini, 549

prebiotic index, 1167

preparative thin-layer

chromatography, 713

principal component analysis, 693

processing, 37

proliferation, 595

prostate cancer, 645

protoplasts, 921

Psathyrella candolleana, 725

purine compounds, 37

radiocesium, 71

RAW264 macrophage, 469

RAW264.7 macrophages, 825

reactive oxygen species, 595

real-time quantitative

polymerase chain reaction, 301

red fluorescent protein, 921

reference genes, 301

response surface methodology, 47, 311, 955, 1007

rheological properties, 611

Rhizoma gastrodiae extract, rice agroresidues, 537

safety, 401

salicylic acid, 955

Salmonella typhi, 713

scavenging capacity, 817

selenium polysaccharides, 1019

sequence reliability, 1223

size exclusion

chromatography/multiangle

skin substitute, 683

Slovakia, 121

soil $\mathrm{pH}, 121$

solid-state fermentation, 353,

657

somatic compatibility, 291

Sparassis latifolia, 301

ß-carotene/linoleic acid, 1051

Staphylococcus aureus, 469

Stereum rugosum, 1075

Stereum sanguinolentum, 1075

stirred tank bioreactor, 253

stirred tank, 735

stress resistance, 1033

stresses, 909

structure elucidation, 155

submerged cultivation, 253,

735, 1115

submerged culture, 841

successive culturing, 1089

sunflower seed hulls, 537

superoxide $\left(\mathrm{O}_{2}{ }^{-}\right)$radicals, 561 
synnema, 59

Syzygium aromaticum, 267

tacrine, 583

Taiwanofungus camphoratus, 595

target therapy, 783

targeted cancer therapy, 105

temperature, 59

Termitomyces robustus, 193

texture profile analysis, 611

total flavonoid contents, 955

trace elements, 493

Trametes versicolor, 735

transcriptome, 1193
Tremella fuciformis, 207, 921

Trichoderma atroviride, 515

triterpenes, 703

tyrosinase inhibition, 275

tyrosinase inhibitory activity, 865

ulcer index, 805

unsaturated fatty acids, 493

unstructured kinetic model,

1207

UVB irradiation, 429

vascular endothelial growth

factor (VEGF), 645

vector mosquitoes, 725 vitamins, 493

volatile compounds, 693

volatile oil, 207

wild mushrooms, 469

wild-growing mushrooms, 393

wistar rats, 805

wound healing, 683

$\mathrm{X}$-ray, 1123

zinc, 343

$\alpha$-amylase, 703

$\alpha$-glucosidase inhibitory

activity, 865

$\alpha$-glucosidase, 703

$\beta$-catenin, 1043 